\title{
Privilegierung und Ausgrenzung in Bildungsprozessen: Soziale Ungleichheit im Lehramt beforschen
}

\author{
Katarina Froebus ${ }^{\star}$, Susanne Kink-Hampersberger ${ }^{* *}$ und Veronika Wöhrer ${ }^{* * *}$ \\ Zusammenfassung \\ Dieser Beitrag stellt ein Forschungsprojekt vor, in dem gemeinsam mit Studierenden soziale Ungleichheit in \\ Schule und Universität beforscht wird. Die Auseinandersetzung mit der Reproduktion sozialer Ungleichheit \\ in Bildungsinstitutionen dient dazu, Habitusreflexivität zu entwickeln und das eigene Handeln als Lehrende \\ hinterfragen und reflektieren zu können. Die Mechanismen der Privilegierung und Ausgrenzung in Bildungs- \\ prozessen werden sowohl auf der Ebene der eigenen Erfahrungen als auch in Auseinandersetzung mit Theorien \\ zu sozialer Ungleichheit zugänglich gemacht und in eigene Forschung übersetzt. Im Fokus des vorliegenden Bei- \\ trags stehen entsprechend Erfahrungen der Studierenden mit Kategorien sozialer Differenzierung und die Frage \\ danach, wie ausgehend von der Einsicht in die strukturelle Dimension sozialer Ungleichheit Reflexionsprozesse \\ angestoßen werden können.
}

Schlagwörter: Habitusreflexivität, Soziale Ungleichheit, Lehramt

\section{Privilege and exclusion in educational practices: Research on social inequality in teacher education}

\section{Abstract}

This article presents a research project in which research about social inequality in schools and universities is done together with students. The aim of this project is to examine the reproduction of social inequality in educational institutions in order to develop habitus reflexivity and to question and reflect on one's own actions as a teacher. Privileging and exclusionary mechanisms in educational processes are made accessible on the level of students' experience as well as in the context of theories of social inequality, and translated into one's own research. Accordingly, the paper focuses on students' experiences with categories of social differentiation and looks for possibilities to initiate reflective processes based on insights into the structural dimension of social inequality.

Keywords: habitus reflexivity, social inequality, teacher education

\footnotetext{
${ }^{*}$ Katarina Froebus, Universität Graz, Institut für Pädagogische Professionalisierung. E-Mail: katarina.froebus@uni-graz.at

** Susanne Kink-Hampersberger, Universität Graz, Institut für Pädagogische Professionalisierung.

E-Mail: susanne.kink@uni-graz.at

***Veronika Wöhrer, Universität Wien, Institut für Soziologie.E-Mail:veronika.woehrer@univie.ac.at

Dieser Artikel basiert u.a. auf dem Vortragstext „Privilegierung und Ausgrenzung in Bildungsprozessen - Soziale Ungleichheit im Lehramt" zum Momentum Kongress 2018 "Klasse“, welcher von den Autorinnen gemeinsam mit Klara Strausz vorbereitet wurde. Seit Mai 2019 wird das Projekt "Habitus.Macht.Bidlung - Transformation durch Reflexion" über die universitäre Leistungsvereinbarung von der Uni Graz gefördert.
}

2019 | innsbruck university press Innsbruck

Momentum Quarterly I ISSN 2226-5538 I momentum-quarterly.org

Vol. 8, No 3 I DOI 10.15203/momentumquarterly.vol.no3.p116-130 


\section{Habitus.Macht.Bildung - Einleitung}

Bildungsinstitutionen sind von einem Mittelschichtshabitus geprägt (Bourdieu 2018). Es verwundert daher nicht, dass Lehrer_innen eine entscheidende Rolle in der (Re-)Produktion sozialer Ungleichheit im Bildungssystem einnehmen. Für angehende Lehrer innen ist es deshalb von zentraler Bedeutung, sich bereits während der Ausbildung mit der eigenen sozialen Herkunft, dem eigenen Habitus und generell der Problematik von sozialer (Bildungs-) Ungleichheit auseinanderzusetzen. Dies erweist sich für sie in doppelter Hinsicht als vorteilhaft, denn sie sind einerseits als Studierende selbst mit der (fehlenden) Passung ihres Herkunfts- mit dem Fachhabitus an der Universität konfrontiert, andererseits beeinflusst ihr eigener Habitus unbewusst die Bewertung ihrer Schüler_innen als begabt, engagiert, bildungsnah - oder auch als unbegabt, faul und bildungsfern. Die symbolische Gewalt der Schule drückt sich nicht nur in den vermittelten Inhalten und der Art der Vermittlung aus, sondern auch darin, dass Kindern unterschiedliche Perspektiven mittels Förderung und Übergangsempfehlungen zuteil werden (Erler 2011; mehr zur symbolischen Gewalt siehe folgender Abschnitt).

Dies zum Anlass nehmend wird im Forschungsprojekt Habitus.Macht.Bildung - Transformation durch Reflexion, der Einfluss sozialer Ungleichheit auf Bildungswege, auf den Universitätszugang und den Verlauf des Universitätsstudiums mit Lehramtsstudierenden thematisiert und erforscht. In einer reflexiven Auseinandersetzung mit habituellen Orientierungen (Helsper 2018: 131) lernen zukünftige Lehrer_innen, diese zu reflektieren bzw. zu hinterfragen und gewinnen dabei Wissen über (implizite) Mechanismen von Privilegierung und Ausgrenzung in Bildungsinstitutionen. Mittels diverser Übungen und eigener Forschungen sollen Lehramtsstudierende scheinbare Automatismen von Privilegierung und Ausschluss insbesondere in Bildungsinstitutionen wie Schulen und Universitäten erkennen und kritisch reflektieren, um so auf längere Sicht Transformationsprozesse anzustoßen.

Der Anspruch des Projektes besteht folglich nicht nur darin, Lehramtsstudierenden auf einer theoretischen Ebene Wissen über pädagogische Forschung zur sozialen Ungleichheit zu vermitteln. Vielmehr geht es uns darum, die eigenständige Auseinandersetzung mit sozialen und besonders in pädagogische Kontexte eingebetteten Machtverhältnissen anzuregen. Dadurch können sich angehende Lehrer_innen vorherrschende Erklärungszusammenhänge in Bezug auf Privilegien oder Ausgrenzung im Bereich der Bildung bewusst machen, pädagogische Zielvorstellungen sowie Haltungen überprüfen und somit neue Perspektiven auf ihr pädagogisches Handeln entwickeln. Ziel des Projekts ist es darüber hinaus, die erprobten Methoden weiter auszuarbeiten, um auch anderen Lehrenden Materialien zur Verfügung zu stellen, die zu einer nachhaltigen Reflexion in Bezug auf soziale Ungleichheit und zur Transformation von Selbst- und Weltverhältnissen beitragen.

Der vorliegende Artikel widmet sich den zugrunde liegenden theoretischen und empirischen Überlegungen des Projekts Habitus.Macht.Bildung und erläutert damit jenes Setting näher, welches Habitusreflexivität angehender Lehrer_innen ermöglichen soll. In den folgenden Kapiteln werden dafür zunächst die theoretischen und methodologischen Hintergründe und damit der Zusammenhang von Habitus, Macht und Bildung (2) dargelegt, darauf folgend werden die empirische Umsetzung und die damit verbundenen Herausforderungen eines partizipativen Lehr-Forschungsprojekts (3) beleuchtet.

Anschließend werden erste Erkenntnisse (4) aus dem Projekt näher erläutert, bezogen auf die Frage, wie Studierende bestimmte Kategorien sozialer Ungleichheit - konkret Geschlecht und Klasse - aufgreifen und verhandeln. Zuletzt wird im abschließenden Fazit (5) das Potenzial des Projektes sowie der vorgeschlagenen Vorgehensweise ausgelotet.

\section{Die Verbindung von Habitus, Macht und Bildung - Theoretische Bezüge}

Soziale Herkunft stellt bislang noch immer den größten Faktor in der Vererbung von Bildungsabschlüssen dar, denn „[d]ie Schullaufbahn eines Kindes hängt von der Bildung, dem Beruf und dem Einkommen der Eltern ab“, so der Soziologe Johann Bacher in seiner Untersuchung über den Zusammenhang von sozialer Ungleichheit, Schullaufbahn und Testleistung (Bacher 2006: Seite 99; ähnlich: Statistik Austria 2018). Bildung steht damit in einem engen Zusammenhang mit sozialer Ungleichheit, auch wenn es der Anspruch von Schule ist, ,objektiv' alle gleich zu behandeln. De facto wird jedoch - durch die ungleiche Ausstattung mit für die Schule wichtigen Kapitalien - die Ungleichbehandlung sowohl verschleiert als auch legitimiert. Hinter der formalen Gleichheit entfalten daher, so zeigt der 
soziologische Blick Bourdieus, die Habitusformen der Einzelnen ihre Wirkung und tragen zur Stabilität der Definitionen und Zuordnungen zu sozialen Kategorien bei. Sie leiten als „strukturierte Strukturen, die geeignet sind, als strukturierende Strukturen $\mathrm{zu}$ wirken“ (Bourdieu 1976: 165) unser Handeln an - sie geben vor, was überhaupt denk- und vorstellbar ist.

Der Ausgangspunkt unserer Überlegungen ist daher die beständige Durchsetzungskraft der „Illusion der Chancengleichheit“ (Bourdieu/Passeron 1971). So benannten Pierre Bourdieu und Jean-Claude Passeron in den 1970er-Jahren den Umstand, dass das Bildungssystem zwar vorgibt, Bildungsbeteiligung gleichermaßen zu ermöglichen und erbrachte Leistungen objektiv zu messen, tatsächlich aber soziale Hierarchien reproduziert und sogar verfestigt, indem durch die vermeintliche Objektivität der Leistung und der scheinbar gleichverteilten Chancen die Illusion einer gerechten Auslese erzeugt wird (Schneickert 2013). Vielmehr wird durch Praktiken in Bildungsinstitutionen symbolische Gewalt ausgeübt, indem bestimmten Wissensformen und -inhalten, bestimmten Rede- und Verhaltensweisen usw. der Vorzug gegeben wird; Privilegierung (bzw. den passenden, also homologen Habitus zu besitzen) wird dann allzu schnell als Begabung gedeutet, Deprivilegierung als „Bildungsferne“. Daher ist das Schulsystem für die Aufrechterhaltung von Klassenunterschieden funktional:

„Wenn es eine Institution gibt, welche die Existenz der sozialen Klassen anerkennt, registriert, absegnet und verstetigt, dann ist es das Schulsystem. [...] Die Funktion des Schulsystems scheint darin zu liegen, die Klassen so zu erhalten, wie sie sind, oder genauer: den Abstand zwischen den Klassen über alle Veränderungen hinweg konstant zu halten." (Eribon 2017: 182f.)

In Anschluss an Bourdieu (1997) lässt sich die Beständigkeit der Verhältnisse, „wie sie sind“, über das Konzept des Habitus erklären. Erworben in klassenbezogenen Sozialisationsprozessen zeigen sich im Habitus als Ausdruck einer inkorporierten Sozialstruktur spezifische Grundorientierungen, Weltansichten sowie Wahrnehmungs- und Bewertungsschemata. Hubert Knoblauch (2003: 188) bezeichnet den Habitus demensprechend als „Einfallstor der Gesellschaft ins Subjekt“. Durch die Auffassung, dass sich der Habitus entsprechend der sozialen Lage, der Geschlechtszugehörigkeit, der Religion oder der ethnischen Zugehörigkeit etc. (Brandes 2002: 8off.) formiert, erweist sich Bourdieus Konzept als anschlussfähig für intersektionale Debatten, kommt doch im Habitus die Verschrän- kung sozialer Kategorien - Bourdieu selbst bezieht sich insbesondere auf Klassen-, Geschlechter- und rassistische Verhältnisse - zum Ausdruck (Hofbauer/ Krell 2011). Im Anschluss daran fassen wir den Habitus als ein „mehrdimensionales Konzept [...], das Dispositionen des geschlechtlich strukturierten Habitus mit jenen etwa des klassenspezifisch, milieuspezifisch oder alters- und generationsspezifisch strukturierten Habitus relationiert" (Hofbauer 2004: 46). Ungleichheitsgenerierende Kategorien werden daher anschließend an intersektionale Debatten in ihren Wechselwirkungen betrachtet (Crenshaw 1989; Klinger 2003; Winker/ Degele 2009) und unter sozialer Ungleichheit wird generell der ungleiche Zugang zu sozialen Positionen verstanden, der systematisch mit vorteilhaften oder nachteiligen Handlungs- und Lebensbedingungen behaftet ist (Solga et al. 2009: 15).

In Bezug auf das Schulsystem zeigt sich die Wirkung des Habitus vor allem darin, dass Schule einer bürgerlichen Idee von Bildung im Sinne des humanistischen Ideals anhängt und dass Lehrer_innen unbewusst auch das Passungsverhältnis von Schüler_innen-Habitus und institutionellem Habitus bewerten. Dabei gerät die Geschichte der körperlichen Aneignung von Bildung, z. B. die körperliche Ruhe des Lesens oder die „Ruhigstellung“ des Körpers in der Schulstunde, allzu leicht aus dem Blickfeld. Durch den Fokus auf intellektuelle Tätigkeiten und den Rückgriff auf die Erzeugnisse bürgerlicher Kultur kommt der schulische Habitus damit besonders jenen Kindern entgegen, „die mit einem homologen Habitus eintreten [...]", während „die schulische Praxis solche Kinder weiter [restringiert], die bereits in der primären Sozialisation von symbolischen Kompetenzen abgeschnitten worden sind“ (Liebau 2011: 16). Eribon (2016) beschreibt eindrücklich, wie die symbolische Gewalt des schulischen Curriculums seine Schulerfahrungen prägte - wenn z. B. im Musikunterricht „Bürgerkinder" beim Vorspielen von klassischen Musikstücken schwärmerische Mienen aufsetzten, während Arbeiter_innenkinder kicherten und schwatzten. „All das trägt insgeheim dazu bei, dass jenen, denen es ohnehin schon schwerfällt, den sozialen Anforderungen des Schulbetriebs in allen seinen Aspekten zu genügen, das Gefühl gegeben wird, sie gehörten nicht dazu und seien dort irgendwie fehl am Platz." (ebd.: 160)

Sowohl die Abläufe als auch die Ziele und Inhalte der Schule wirken in ihrer Willkürlichkeit (Liebau 2011: 19) selbstverständlich, aber über sie wird symbolische Gewalt ausgeübt. „Mit symbolischer Gewalt 
meint Bourdieu Formen ,gewaltloser Gewalt', die dafür sorgen, dass Unterprivilegierte ihre subalterne Position anerkennen, obwohl sie dadurch offenkundig benachteiligt werden" (Erler 2011: 26). Diese Form von Gewalt, für die Bourdieu synonym auch die Begriffe symbolische Macht oder symbolische Herrschaft verwendet, wird über Kultur, Sinnbezüge, Weltansichten und selbstverständliche Denkweisen vermittelt und daher im öffentlichen Bildungswesen, in Kirchen, Medien, Kunst und Literatur sowie den Wissenschaften aktualisiert und verbreitet (Moebius/Wetterer 2011: 1f.). Symbolische Gewalt schreibt sich in den Körper ein und wird als solche nicht greifbar, weil „sie auf der symbolisch-sinnhaften Ebene des Selbstverständlichen und Alltäglichen operiert und zur Bejahung, Verinnerlichung und Verschleierung von gesellschaftlichen Herrschaftsverhältnissen führt" (ebd.: 1). Spürbar wird sie beispielsweise in der Scham und der Selbstexklusion der Bildungsbenachteiligten. Auch in Bildungsprozessen wird die „soziale Grammatik“ erlernt, wird der Habitus im Körper verankert und die soziale, geschlechtliche oder ethnische Zugehörigkeit „inkorporiert“. Die Aneignung spezifischer Wahrnehmungs-, Denk- und Handlungsmuster geschieht nicht explizit, sondern auf der Ebene impliziter Erziehung. Da der Konflikt um soziale Zugehörigkeiten nicht offen ausgetragen wird, besteht die Herausforderung darin, Erfahrungen symbolischer Gewalt für Lehramtsstudierende überhaupt erst wahrnehmbar $\mathrm{zu}$ machen. Das Trügerische ist dabei die (scheinbare) Individualität dieser Erfahrungen, die sowohl auf der Seite der Privilegierten als auch der Unterprivilegierten die Deutung eigener Erfahrungen bestimmt. Auch die Einschätzung des eigenen Schul- und Studienerfolgs wird daher durch soziale Positionen beeinflusst. Gute Leistungen werden je nach Positionierung der eigenen Begabung zugeschrieben, als Ergebnis fleißigen Lernens verstanden oder als unwahrscheinlicher Zufall aufgefasst (Kramer 2013: 15). Deutlich ist jedoch, dass trotz unzähliger bildungspolitischer Bemühungen ( $\mathrm{z}$. B. Inklusion oder Kompetenzorientierung) und vielfältiger Maßnahmen (wie beispielsweise Gleichstellungsprogramme oder (Mentoring-)Programme, die Studierende aus Nicht-Akademiker_innenhaushalten unterstützen), Lehren und Lernen auch heutzutage noch immer von Hierarchien und Ungleichheit bzw. Ausschluss und Privilegierung durchzogen sind.

Da der Habitus durch das Aufwachsen in einer sozialen Gruppe erworben wird, gibt dieses Aufwachsen den „Möglichkeitshorizont“ vor und bestimmt so auch das Verhältnis zu den „herrschenden Verhältnissen“, den Umgang mit sozialen Unterschieden sowie die Anerkennung der Legitimität von Ungleichheit (Liebau 2014: 156). Es ist daher auch eine Konsequenz aus einer niedrigen sozialen Positionierung, der Illusion der Chancengleichheit zu erliegen. Selbst wenn deutlich zutage tritt, wie das Bildungssystem Ungleichheit systematisch reproduziert, so bleibt weiterhin problematisch, dass Bildungsbenachteiligte ihre Interessen nicht durchsetzen können (Castro-Varela 2014). Der Klasse der Beherrschten gehören jene Personen an, welche über zu wenig Kapitalvolumen verfügen, um es in Titel und Abschlüsse konvertieren zu können. Durch ihre Vergabe von Bildungsabschlüssen und Titeln sichern Bildungsinstitutionen so die Privilegien der herrschenden Klasse. Bildung ist demzufolge kein Mittel zur Aufhebung sozialer Ungleichheit, sondern trägt zur Stabilisierung von Herrschaft bei (Bourdieu 2018; Heim/Lenger/Schumacher 2009: 254). ${ }^{1}$ Deutlich wird so, dass die (bildungspolitische) Forderung nach Gleichheit Ungleichheit sowohl zur Voraussetzung als auch zum Ziel hat. Welche pädagogischen Konsequenzen lassen sich dann aus der Einschätzung Kollers, „dass das Aufwachsen von Kindern und Jugendlichen weit mehr von der gesellschaftlich ungleichen Verteilung von Kapital abhängt als vom erzieherischen Handeln von Pädagoginnen und Pädagogen“ (Koller 2009: 154), für Lehramtsstudierende ziehen?

Die Soziologie Bourdieus ruft nicht nur zu einer Kritik an machtvollen Über- und Unterordnungsverhältnissen auf - denn im Sinne einer reflexiven Soziologie gilt es nicht nur „die Funktionsweise symbolischer Gewaltverhältnisse zu erforschen" und damit die „Praktiken der Legitimierung und Modi der Inkorporierung “ in den Blick zu nehmen, sondern ebenso die „eigene Involviertheit in diesen Praktiken“ zu reflektieren (Alkemeyer/Rieger-Ladich 2008: 104) ${ }^{2}$ und damit den eigenen Habitus reflexiv zu betrachten.

1 Dieser Befund gilt ebenso für Österreich, wie beispielsweise die von Statistik Austria erhobenen Zahlen zur Vererbung von Bildung (Statistik Austria 2015), der Nationale Bildungsbericht (Mayrhofer et al. 2019, besonders Teil „C2 Segregation bei Schulwegentscheidungen“), die Zahlen der OECD Studie (OECD 2018) oder die Untersuchung von Breit und Schreiner (2017) verdeutlichen.

2 Bourdieu selbst hat konkret in Form einer „reflexiven Pädagogik" den Pluralismus von Leistungsformen gefordert und sich gegen die Privilegierung sprachlicher Kommunikation sowie abstrakter Wissensformen ausgesprochen (Rieger-Ladich 2017: 351) 
Die Lehrer_innenbildung an der Universität steckt hier aber in einem doppelten Dilemma, denn einerseits vertritt und reproduziert sie das Kritisierte, soll aber andererseits auch Studierende dazu anregen, eigene, kritische Positionierungen hervorzubringen (vgl. hierzu auch Hermann 2016). Auch Lehramtsstudierende erleben an der Universität mitunter die fehlende Passung ihres Habitus mit dem Fachhabitus. Sie müssen sich in (auch disziplinär unterschiedliche) Zugänge zu Wissenschaft einfinden, die aus zunächst meist unverständlichen Codes und Regeln bestehen, in welche sie erst hineinsozialisiert werden. Trotz der Herausforderungen durch das Studium vertrauen Studierende dem Leistungsprinzip - andere, neue Erklärungsmuster müssen erst etabliert werden: „Um einen Zugang zu dem Leiden an dem System Schule zu bekommen“ ist es daher für angehende Lehrer_innen von zentraler Wichtigkeit, „soziale Selektivität nicht zu befördern“, es bedarf stattdessen „eines Zugangs zu den unbewussten, habituell geprägten Verstrickungen“ (Vogel 2019: 233). Die eigenen Erfahrungen des Bildungsaufstiegs und die langsame „Habitustransformation“" (El-Mafalaani 2017) im Laufe des Studiums, die sich vor allem durch den Erwerb von kulturellem Kapital und der Aussicht auf ein zukünftig höheres ökonomisches Kapital vollzieht, können zum Ausgangspunkt von Reflexionsprozessen gemacht werden: Was bedeutet es ganz konkret, sich in Bildungsinstitutionen mehr oder weniger willkommen oder fremd zu fühlen? Welche Erlebnisse haben die Studierenden selbst gemacht?

\section{Habitusreflexion mit Lehramtsstudierenden - Empirische Umsetzung des Projektes}

Ausgehend von der Annahme, dass angehende Lehrer_innen an der Universität den gesellschaftlichen Kräfteverhältnissen auf eine bestimmte Art und Weise ausgesetzt sind, setzt das Projekt Habitus. Macht.Bildung daher an der Habitusreflexion sowie den durchlebten Erfahrungen von Privilegierung und Diskriminierung der Lehramtsstudierenden an. Zum Thema werden insbesondere die eigene Bildungsbiografie, Herkunft und zur Verfügung stehende Ressourcen gemacht, um so die persönliche Verortung im sozialen Raum beforschen zu können. Damit stehen die Erkundung des individuellen Habitus in Verbindung mit gesellschaftlichen Strukturen und die Reflexion der eigenen Involviertheit hinsichtlich legitimierender Praktiken und Inkorporierungsmodi im Zentrum des
Interesses. Die Reflexion der eigenen Erfahrungen mit sozialer Differenzierung an Bildungsinstitutionen soll dazu beitragen, diese als Resultat von Machtverhältnissen und Kämpfen um Hegemonie verstehen zu lernen. Im Sinne einer reflexiven Erziehungswissenschaft (Friebertshäuser et al. 2006) wird also der Habitus in Bezug auf die eigenen Erfahrungen mit Privilegierung und Ausschluss innerhalb der Universität (aber auch Schule) thematisiert. Eine selbstreflexive, machtanalytische und dekonstruktive Auseinandersetzung mit sozialer Ungleichheit birgt dabei für Lehramtsstudierende das Potenzial, in Bezug auf das künftige eigene Lehren an einer Bildungsinstitution emanzipatorische Handlungsperspektiven zu entwickeln, die z. B. das Aufbrechen bisheriger Wahrnehmungs- und Handlungsmuster im Umgang mit Schüler_innen und bei der Bewertung ihrer Leistungen bewirken können.

Aufbauend darauf ist das Vorgehen im Projekt so angelegt, dass in einem ersten Schritt im Rahmen der Lehrveranstaltung „Einführung in die pädagogische Forschung $^{\text {“3 }}$ mit Studierenden der Einfluss sozialer Ungleichheit auf Bildungswege, auf den Universitätszugang und im Verlauf des Universitätsstudiums thematisiert wird und anhand verschiedener Übungen Reflexionsprozesse eigener habitueller Orientierungen Orientierungen angeregt werden. Die Studierenden lernen in diesem Setting, dass Bildung und Bildungsinstitutionen in Analogie zu bestehenden Herrschaftsverhältnissen der Gesellschaft konstituiert werden. Darüber wird sichtbar, dass diese Verhältnisse auch die konkrete Formulierung und Durchsetzung von Forschungsinteressen durchdringen. Konkret arbeiten wir in dieser Lehrveranstaltung ${ }^{4}$ mit einer Mischung aus theoretischen Inputs (etwa zu Bildungsungleichheit, der sozialen Herkunft von Lehrer_innen, zu Wissenschaft als sozialem Feld etc.) sowie Methoden der Biografiearbeit, der qualitativen Sozialforschung und der partizipativen Forschung. Zum Einsatz kommen dabei Kollektive Erinnerungsarbeit (Haug 1999; Ortner/Thuswald 2012), leitfadengestützte und narrative Interviews (Bock 1992; Rosenthal/Loch 2002), Gruppendiskussionen (Bohnsack et al. 2006), Photovoice (von Unger 2013) sowie andere partizipative Methoden zur Selbstreflexion wie beispielsweise

3 Bachelorstudium Lehramt Sekundarstufe Allgemein, Modul BWB Bildungstheorie und Gesellschaft.

4 Diese wird seit dem Sommersemester 2018 in jeweils zwei parallelen Lehrveranstaltungsgruppen in jedem Semester von uns geleitet. 
schriftliche Reflexionen über die eigenen Positionierungen zur Wissenschaft (Costa/Mendel 2018) oder Visualisierungen der jeweils zur Verfügung stehenden Ressourcen und Kapitalsorten (Bourdieu 1983).

Forschung und „Selbstbeforschung“ der Studierenden finden sowohl im Rahmen von kleineren Übungen zur Selbstreflexion als auch größeren Projekten statt. Besonders Übungen wie „Differenzbingo", die Verbildlichung der eigenen Ausstattung mit Kapital oder Diskussionen darüber, ob es einen spezifisch studentischen Habitus (und besonders Geschmack) gibt, thematisieren sehr deutlich Unterschiede und können direkt in der Gruppe eingesetzt werden. Spannend ist es aber auch, die theoretische und persönliche Auseinandersetzung mit sozialer Ungleichheit in eigene Forschungsfragen $\mathrm{zu}$ übersetzen. Dafür müssen sich die Studierenden grundlegend mit Methoden qualitativer Forschung beschäftigen. Während eine Lehrveranstaltungsgruppe überwiegend mit der Methode der Erinnerungsarbeit arbeitet, nähert sich die andere Gruppe von Studierenden der Thematik sozialer Ungleichheit mit eher klassischen qualitativen und partizipativen Methoden und führt Gruppendiskussionen bzw. Interviews mit Studienkolleg_innen aus dem Lehramt durch und nutzt die Photovoice-Methode.

Die Erinnerungsarbeit verbindet Elemente der partizipativen und der qualitativen Forschung auf eine eigene Art: Hier wird die Unterscheidung zwischen Forschenden und Beforschten hinfällig, weil die Forschenden gleichzeitig auch ihre eigenen Forschungsobjekte sind. ${ }^{6}$ Mit dieser Verschiebung ver-

5 In den Feldern des Bingozettels stehen Fragen, die mehr oder weniger soziale Unterschiede thematisieren. Wie beim klassischen Bingo geht es darum, eine Reihe vertikal, horizontal oder diagonal auf dem Bingozettel zu füllen, indem Kolleg_innen gefragt werden. Wenn man jemanden findet, der_die beispielsweise eine Privatschule besucht hat oder dessen_deren Eltern nicht in Österreich geboren wurden, darf man diese Kategorie abhaken. Im Anschluss daran wird die Übung gemeinsam im Plenum besprochen und es werden somit Phänomene struktureller Benachteiligung und Privilegierung diskutiert und reflektiert. Für Beispiele siehe z. B. https://www.rosalux.de/fileadmin/rls_uploads/pdfs/Bildungsmaterialien/heft4/methode_01.pdf [05.04.2019].

6 Die Methode wurde von einer Gruppe deutscher „kritischer Psychologinnen“ rund um die Psychologin und Soziologin Frigga Haug entwickelt: In der Erinnerungsarbeit verschmelzen Subjekt und Objekt der Forschung sowie der Anspruch der Forschung mit dem der Selbstveränderung. Anhand von "Erinnerungen" zu einem bestimmten Thema, die als Geschichten in der dritten Person aufgeschrieben bindet sich das Ziel, die eigene Handlungsfähigkeit zu vergrößern, indem gesellschaftliche Bedingungen und strukturelle Gemeinsamkeiten individueller Erfahrungen näher beleuchtet werden. Die Begründerin der Erinnerungsarbeit, Frigga Haug, legt den Fokus auf das „Entlernen“, also die aktive Arbeit an der Veränderung (Haug 2003). Es geht darum, im Alltäglichen die "herrschenden Verhältnisse" aufzudecken und andere Deutungen und damit Handlungsoptionen sichtbar zu machen. In einer Mischung aus Ideologiekritik und Diskursanalyse werden die Erfahrungen der Einzelnen als diskursiv geformte verstanden und damit unser alltägliches Wissen als ideologisch „enttarnt“ (vgl. dazu auch Hipfl 1997; Brandmayr 2017). Aus den Analysen kollektiver Erfahrungen durch die Arbeit mit Erinnerungsgeschichten der Studierenden können neue Einsichten über die Macht gesellschaftlicher Normalvorstellungen und eigener Ansprüche entstehen und es zeigt sich, dass Erfahrungen des Zweifelns und der Unsicherheit von vielen geteilt werden. Zentral ist bei der Erinnerungsarbeit auch, dass der Impulssatz für die Erinnerungsgeschichte gemeinsam gefunden werden muss, d. h. es ist ein intensiver Austausch in der Gruppe nötig, um sich auf einen gemeinsamen Ausgangssatz (z. B. "Als ich an der Uni einmal auf mich allein gestellt war") zu einigen.

Dieses gemeinsame Finden einer Thematik, in diesem konkreten Fall der Forschungsfrage und der gemeinsamen Erarbeitung der Leitfäden, wird in der Parallelgruppe der Lehrveranstaltung zu einem zentralen Moment. Wie in der anderen Lehrveranstaltungsgruppe geht es auch hier um die Wahl gemeinsamer Themen, die die Studierenden in Bezug auf soziale (Bildungs-)Ungleichheit als wichtig empfinden, und

werden, setzt sich eine Gruppe von Frauen mit persönlich Erlebtem, dessen Verarbeitung und Rekonstruktion auseinander. Die Geschichten werden kollektiv diskutiert und analysiert, auf Brüche, Auslassungen und Harmonisierungen hin untersucht. Es wird herausgearbeitet, wie sich gesellschaftliche Muster in diese Geschichten einschreiben bzw. wie sich Frauen in ihren konkreten Lebenspraxen objektive Ordnungsstrukturen subjektiv aneignen und sich in diesem Vergesellschaftungsprozess selbst aktiv herstellen. Die Geschichten werden also als subjektive Realitätsverarbeitung, -verzerrung und -verdrängung zwecks Konstruktion eines akzeptablen Selbst aufgefasst. Brüche und Leerstellen dienen als Ausgangspunkte für ein Neuschreiben der Geschichten, die dann erneut analysiert werden - so wird der persönliche und gesellschaftliche Handlungsspielraum reflektiert und erweitert (Haug 1999, Ortner/Thuswald 2012, Bitschnau/Hinteregger 2001) 
um die Formulierung konkreter Fragen, mittels derer der Thematik nachgegangen wird. Die Studierenden werden hier zwar nicht immer selbst zum Gegenstand der eigenen Forschung, jedoch - etwa im Rahmen der Photovoice-Methode ${ }^{7}-$ im Sinne einer partizipativen Vorgehensweise zu Ko-Forscher_innen. ${ }^{8}$ Forschung wird in diesem Lehrveranstaltungssetting als ein wechselseitiges Lernen von Forscher_innen und Erforschten begriffen, anstatt sie als Herrschaftsbeziehung zu erleben (Aulenbacher et al. 2010: 95). Durch die vergleichsweise große Offenheit begünstigen etwa Gruppendiskussionen und vor allem narrative Interviews ein gegenseitiges Reagieren aufeinander. Dies äußert sich beispielsweise darin, dass von den Interviewten im Vergleich zum zuvor gemeinsam konstruierten Leitfaden ${ }^{9}$ in den Interviews oder Gruppendiskussionen andere Bereiche sozialer Ungleichheit aufgegriffen wurden. Untersuchungspersonen werden somit durch das Zulassen der eigenen Erzählungen nicht zum Objekt der eigenen Forschung degradiert, sondern als „Subjekte mit eigenen Relevanzstrukturen“ (Müller 1984: 33f.) ernstgenommen. Darüber hinaus soll die Bearbeitung mit klassisch qualitativen Ansätzen den Blick der Studierenden für strukturelle Bedingungen sozialer Ungleichheit abseits individueller Erfahrungen anregen und schärfen.

7 Die Photovoice-Methode ist eine der bekanntesten partizipativen Methoden (u. a. von Unger 2013), bei welcher Bilder als Daten verwendet werden. Entlang einer Fragestellung, die von Ko-Forschenden und Wissenschaftler_innen gemeinsam erarbeitet wird, sollen über einen bestimmten Zeitraum Fotos gemacht werden. Diese werden dann in einer Gruppendiskussion oder einem Interviewsetting gemeinsam diskutiert und in Form einer „Ausstellung “ präsentiert.

8 In der partizipativen Forschung wird nicht „über" oder „für" Menschen geforscht, sondern „mit" ihnen, wie Bergold und Thomas (2010) betonen. D. h. statt "beforscht" zu werden, arbeiten die Personen, die von einer Fragestellung betroffen sind, direkt an der Forschung mit, ohne ausgebildete Sozialwissenschaftler_innen sein zu müssen.

9 Für die Durchführung der narrativen Interviews erarbeiten die Studierenden einen gemeinsamen Leitfaden, der sie als „Neulinge“ in der Interviewführung unterstützen soll. Dabei wird im Rahmen der Lehrveranstaltung eine gemeinsame Forschungsfrage erarbeitet sowie eine gemeinsame Einstiegsfrage, die die Interviewten zu einer möglichst offenen Erzählung in Hinblick auf den eigenen Bildungsweg und darin vorkommende Diskriminierung oder Privilegierung anregen soll. Des Weiteren erarbeiten die Studierenden Thematiken sozialer Ungleichheit, die stichwortartig auf dem Leitfaden notiert werden, um in der auf die Eingangserzählung folgenden Phase hier konkrete Anhaltspunkte zur Nachfrage parat zu haben.
$\mathrm{Ob}$ das Durchführen von Forschung zu sozialer Ungleichheit darüber hinaus Reflexionsprozesse bei den Lehramtsstudierenden auslöst, können wir nur mutmaßen. Deutlich wird in der Lehrveranstaltung, dass sich die Studierenden sehr stark auf das Thema einlassen, an dem Thema interessiert sind, neugierig in die Forschungen starten und bereitwillig die Übungen zur Selbstreflexion mitmachen. Die Notwendigkeit zur Auseinandersetzung mit dem eigenen Habitus scheint für Lehramtsstudierende unmittelbar einleuchtend. Gleichzeitig bringen sie oft wenig theoretisches und methodisches Vorwissen mit - hier besteht die Herausforderung darin, die Lehrveranstaltung nicht im Versuch, alle Lücken zu füllen und allen spannenden Fragen nachzugehen, zu überfrachten. Einerseits steht im Rahmen der Lehrveranstaltung „Einführung in die pädagogische Forschung" die Vermittlung von pädagogischen Forschungsmethoden im Fokus, andererseits erheben wir als Lehrveranstaltungsleiter_innen aber auch den Anspruch, diese konkret am Thema sozialer Ungleichheit $\mathrm{zu}$ erlernen, wenngleich den Studierenden diese Thematik oft erst theoretisch nähergebracht werden muss. Diese Diskrepanz zeigt sich beispielsweise dann deutlich, wenn von den Studierenden in den Interviews Fragen formuliert werden, die Stereotype und Klischees - etwa in Bezug auf die Geschlechterthematik, wenn nach Unterschieden zwischen männlichen und weiblichen Lehrkräften gefragt wird - wieder reproduzieren, statt zu hinterfragen und aufzubrechen. Jedoch erkennen die Studierenden in der gemeinsamen Auswertung des Datenmaterials anhand ganz konkreter Beispiele die Illusion der Chancengleichheit im österreichischen Bildungswesen und erfahren Stärkung darin, dass ihre erlebten Erfahrungen nicht nur sie selbst als Individuen betreffen, sondern tief verwurzelt in den strukturellen Gegebenheiten sind. Die Arbeit mit der Erinnerungsarbeit hingegen setzt bei den Lehramtsstudierenden eine gewisse Affinität zum „Zerlegen“ von Sprache voraus. Ihnen steht zwar ein detaillierter Analyseleitfaden zur Verfügung, allerdings werden die Schritte im fortlaufenden Analyseprozess immer komplexer. Gerade die Frage nach dem Einfluss von gesellschaftlichen Diskursen und Wertvorstellungen auf die in den Texten vorhandenen Subjektkonstruktionen bleibt oft unbeantwortet. Besonders hier zeigt sich aber, dass die Vorgehensweise der schrittweisen Dekonstruktion von Bedeutung Gefühle der Unsicherheit, Angst und Scham „herausschälen“ kann, die hinter einer Rhetorik verborgen liegen, in der gesellschaftlichen Erwartungen und Normvorstellungen entsprochen wird. 
Im Rahmen der Lehrveranstaltung werden den Studierenden also nicht nur theoretische Konzepte und sozialwissenschaftliche Methoden vermittelt, sondern sie lernen sozialwissenschaftliches Forschen aktiv kennen, indem sie selbst Forschung betreiben. Zugleich wird ihnen der Raum geboten, über ihre eigene Bildungslaufbahn, universitäre Sozialisation und ihren Habitus nachzudenken. Die dabei gemachten Erfahrungen und gesammelten Daten sind für die Lehrveranstaltungsleiter_innen auch über die konkrete Lehre hinaus interessant und werden im Rahmen des Projekts analysiert und interpretiert.

\section{4. „Wenn du dich nicht einfügen kannst, kannst du's nicht und fertig" - Erste Erkenntnisse}

Ausgehend vom aktuell vorliegenden Datenmaterial wollen wir nun einen Einblick in erste gewonnene Erkenntnisse geben. Strukturiert wird diese Darstellung durch die Frage, wie bestimmte Kategorien sozialer Ungleichheit - im Konkreten Geschlecht und Klasse - in der Lehrveranstaltung aufgegriffen und verhandelt werden. Abschließend loten wir das emanzipatorische Potenzial der Methoden und Übungen aus. Damit Habitusreflexion konkret werden kann, werden Diskriminierung und Privilegierung als Ausdruck struktureller Ungleichheit verhandelt, anstatt diese als rein individuelle Problematik zu verstehen.

\subsection{Diskriminierung - kein Thema an Universi- täten?}

Bei der ersten Durchsicht der Forschungsmaterialien wird deutlich, dass den Studierenden zunächst die Erfahrung der Universität als anonymer Instanz gemeinsam ist, in der das eigene Handeln kaum Einfluss auf das Schicksal hat.

„Es war nicht so die Wärme von einem engen Verband, sondern das war anonym zum größten Teil. Also wie so ein kleiner Fisch in einem ganz großen Meer. Also ich habe nicht wirklich so übertriebene Signale gespürt, dass ich da jetzt reinpasse. Es muss sich quasi jeder sein Glück selbst zusammensuchen."(SU1_08: 24-26 ${ }^{10}$ )

10 Die von den Studierenden geführten Interviews wurden zur Analyse in MAXQDA überführt und mit Nummern versehen. Diese sind, wie am Beispiel SU1_08: 24-26 ersichtlich, folgendermaßen aufgebaut: $\mathrm{SU}=$ Soziale Ungleichheit, $1=$ erster Durchgang (SoSe 2018), 08 = Interviewnummer, 24-26 Zeilennummer im Interview. Zitate aus den Reflexionen, aus den Gruppendiskussionen und den
In der Überzeugung, dass jede_r es schaffen kann, setzt sich der Glaube an das meritokratische Prinzip der Schule auch an der Universität fort: „[...] einen Nachteil habe ich eigentlich nicht gehabt, und ich bin auch nicht benachteiligt worden, weil ich finde, auf der Uni werden alle gleich behandelt. Wenn du dich nicht einfügen kannst, kannst du's nicht und fertig." (SU2_O1: 264-266) Was hier allerdings auch deutlich wird, ist der Ausschluss derjenigen, die sich nicht einfügen können. Der Gleichheitsdiskurs gilt nicht für alle.

Fälle von konkreter Diskriminierung, insbesondere an der Universität, werden daher nur sehr selten berichtet, wenngleich die Durchsicht des Materials und der eigenen Reflexionsprotokolle zeigt, dass sie vorhanden sind. So äußern sich Studentinnen im Rahmen einer gemeinsamen Kodierübung von Interviews dahingehend, dass sie in ihrem Fach der Mathematik "nur“ als Lehramtsstudierende akzeptiert werden, denn nach Ansicht eines Professors würden sich Frauen für „richtige“ Mathematik nicht eignen. Diese Art der auf das Geschlecht bezogenen Diskriminierungserfahrung ist Teil einer umfassenderen Diskriminierung, die Lehramtsstudierende allgemein erleben, weil sie Lehrveranstaltungen gemeinsam mit Studierenden anderer Bachelor-Studien besuchen. Immer wieder bekommen sie nämlich zu hören, dass es für sie als Lehramtsstudierende im Vergleich zu „richtigen“ Bachelorstudierenden doch um ein Vielfaches einfacher sei. Auch der Fall eines Kommilitonen, der ob eines fehlenden „richtigen“ Bildungsabschlusses - das wäre die Matura, er selbst hat die Studienberechtigungsprüfung - und der ständigen Thematisierung dieses vermeintlichen Defizits durch den Professor aus einem Kurs regelrecht hinausgemobbt wurde, lässt sich dieser Differenzierung zwischen den „richtigen“ Bachelor-Studierenden und den Lehramtsstudierenden zuordnen. Und auch der folgende Interviewausschnitt verdeutlicht noch einmal recht anschaulich, dass auch Hochschul-Lehrende stereotype Ansichten und Meinungen vertreten, die Studierende abseits des als normal geltenden Bildungsweges diskriminieren. Eine Lehramtsstudierende berichtet im Zuge eines Interviews etwa von der Meinung einer Lehrenden, dass „wenn man aus einer Arbeiterfamilie kommt, in die Hauptschule und in ein BORG gegangen ist, dass man in seinem Leben zu nichts kommt, dass man auch nicht

Erinnerungsarbeiten sind mit dem Kürzel der Studierenden, das sie sich zu Beginn der Lehrveranstaltung ausgesucht haben, bezeichnet (eine weitere Nummerierung ist bislang nicht erfolgt). 
an die Uni kommt, dass man im Prinzip die Chancen auch nicht hat" (SU2_03: 25-28). Dabei problematisiert sie, dass alle drei Kategorien auf sie zutreffen würden und sie es dennoch auf die Universität geschafft habe. Dies tut sie gegenüber der Lehrperson folgendermaßen kund: „Ich habe sie dann darauf angeredet, weil ich das nicht so toll finde, wenn man die Kategorisierung macht, weil es eben auf mich zutrifft, und sie ist dann der Meinung gewesen: ,Nein, ich bin ein Ausnahmefall $^{\text {““ }\left(\mathrm{SU}_{2} \_3:\right.}$ 32-34). Die Kategorisierung als Ausnahmefall verstärkt sich weiters noch darin, dass dies immer wieder zum Lehrveranstaltungsinhalt wird und die Studierende sich dahingehend abgewertet fühlt:

"Sie hat in fast jeder Einheit, die wir gehabt haben, hat sie eben davon gesprochen, dass eben, irgendwas mit den drei Kategorien, dass eben aus denen nichts werden kann, aber ich bin so dagesessen und hab irgendwie so gedacht: ,Aber Entschuldigung, ich sitze trotzdem noch immer da und ich studiere und ich glaube nicht, dass ich so schlecht bin in dem was ich tu; also, ja. Sie hat mich zwar nicht direkt anders behandelt, aber man hat halt einfach gemerkt, dass sie jetzt einen anderen Blick auf mich hat, weil sie halt einfach weiß, was mein Hintergrund ist." (SU2_03: 70-76)

Im Rahmen der gemeinsamen Besprechung solcher Fallbeispiele zeigt sich jedoch immer wieder, dass die Studierenden dies zuerst als Problem einzelner Individuen behandeln und es eines großen Aufwandes bedarf, um ihren Blick in Bezug auf Privilegierung und Ausschluss basierend auf sozialen Kategorien sowie generell strukturelle Diskriminierung zu schärfen. Es ist daher gleichzeitig überraschend wie auch nachvollziehbar, dass konkrete Diskriminierungserfahrungen kaum zur Sprache kommen. Vielmehr beschreiben die Studierenden erfolgreiche Bildungswege, ihre Leistungsorientierung, das Glück, ihre Interessen an der Universität endlich verfolgen zu können, eine Studienwahl basierend auf den eigenen Kompetenzen oder ihre allgemeine privilegierte Position in der Gesellschaft. Ein Studierender dazu: „Wie sagt man so schön, ich bin auf die Butterseite gefallen. Hab immer alles bekommen, sofern es gegangen ist. Jegliche Unterstützung erfahren, ja, von der ganzen Familie.“ (SU1_19: 153-154)

\subsection{Geschlecht - komplementäre Eigenschaften als Notwendigkeit}

Auch wenn es unbestritten ist, dass Ungleichheit herrscht, scheint trotzdem ein Konsens darüber zu bestehen, dass man selbst nicht davon betroffen ist.
Dies gilt ganz besonders für die soziale Kategorie "Geschlecht". Vielmehr scheinen sich die Studierenden einig zu sein, dass Mechanismen von Privilegierung und Ausschluss aufgrund eines bestimmten Geschlechts an der Universität heutzutage kaum noch wirksam werden. Obwohl einzelne (wenige) Geschichten davon erzählt werden, dass es feministische, aber auch sexistische Professor_innen gäbe, die aus verschiedenen Gründen weibliche Studierende bevorzugten (SU1_o1; sowie Gruppendiskussion im SoSe 2018), oder dass es für männliche Studierende im frauendominierten Bereich der Erziehungswissenschaften leichter sei, Aufmerksamkeit und Unterstützung zu bekommen (SU1_16), stimmen die Studierenden in der Gruppendiskussion überein, dass sie Diskriminierung aufgrund von Geschlecht an der Universität bis dato nicht begegnet seien. Auch im Rahmen der Interviews, die von den Studierenden geführt wurden, werden Diskriminierungserfahrungen aufgrund des Geschlechts nicht als solche benannt. Um dies zu verstehen, wollen wir einen Ausschnitt aus einem Interview genauer darstellen. Eine interviewte Person antwortet auf die binäre Oppositionen reproduzierende - Frage, „ob es da irgendwie einen Unterschied gibt, ob du von einer männlichen Person unterrichtet wirst oder von einer weiblichen" mit folgenden Worten:

„Das ist eine sehr komplexe Frage, weil eine Frau ist nicht gleich eine Frau und ein Mann ist nicht gleich ein Mann. Das sind ja verschiedene Menschen. Das ist ja nicht nur, dass es nur einen Stereotyp gibt, und man kann, glaube ich nicht, Gruppe Männer und Gruppe Frauen auf verschiedene Merkmale reduzieren, weil Merkmale von Frauen auch in der Gruppe Männer vertreten sind und Merkmale, die die Männer haben, in der Gruppe der Frauen vertreten sind. Das heißt, man kann ziemlich schlecht herausfiltern, was ist dann typisch Frau, was ist typisch Mann."(SU1_o8: 383-386)

Auch auf zwei weitere Nachfragen meint die Person zuerst, dass es schwierig sei, zwischen Männern und Frauen allgemein zu differenzieren, ergänzt dann aber in einem Nachsatz, dass Fächer, in denen emotionale Intelligenz eine Rolle spiele, eher von Frauen, aber Technik in Werkstätten eher von Männern unterrichtet werde. Auf die Nachfrage, ob die Person denkt, dass dies naturgegeben sei oder an sozialen Gründen liege, erläutert sie: „Ich glaube schon, dass von Natur aus die Geschlechter unterschiedliche Rollen besser erfüllen können“ und weiter, dass es „für den Fortbestand der Menschheit“ wichtig sei, dass „Frauen gut auf Menschen emotional eingehen können und Männer mehr räumlich-motorische Fähigkeiten haben, um ,Kampf, 
Wegrennen, Konstruieren, bis zum Einparken leisten zu können." (SU1_08: 399-404)

Diese beiden Erklärungsansätze scheinen zunächst sehr unterschiedlich, ja fast gegensätzlich zu sein: Zuerst wird betont, dass eine Einteilung in Männer und Frauen in Bezug auf den Unterricht eigentlich nicht zu beantworten ist, denn dies seien keine homogenen Gruppen und in sich sehr vielfältig. Eine Klassifikation in Männer einerseits und Frauen andererseits sei folglich nicht möglich. Wie dieses Beispiel anschaulich verdeutlicht, wird von der Person gegen geschlechterstereotype Wahrnehmungen und Zuordnungen argumentiert. Knapp darauf werden aber doch Unterschiede thematisiert, die zum einen den klassischen stereotypen Zuordnungen entsprechen und zum anderen als natürlich gegeben begründet werden.

Beide Aussagen haben aber etwas gemeinsam: Sie verdeutlichen, warum die Relevanz von Geschlecht in Bezug auf soziale Ungleichheit für die Studierenden zumeist nicht erfahrbar wird. In der ersten Erklärung werden stereotype Zuteilungen zu Frauen und Männern negiert und damit individuelle Gegensätze betont. Es gehe nicht um das Geschlecht einer Person oder um Eigenschaften innerhalb der Gruppe von Frauen und Männern, sondern vielmehr um individuelle Eigenschaften und Fähigkeiten verschiedener Personen. Daher sei auch das Geschlecht kein Faktor, aufgrund dessen in der Schule oder auf der Universität Leistungen, Rechte oder Ressourcen verteilt werden. In der zweiten Erklärung wird auf Unterschiede zwischen den Geschlechtern verwiesen, die von Natur aus bestehen und die weder hierarchisch noch ungerecht gedacht werden: Sie werden als komplementäre Eigenschaften aufgefasst, die beide notwendig sind, damit die Menschheit überleben könne.

Diese beiden Erklärungsmuster finden sich in vielen Interviews und auch in der Gruppendiskussion wieder. Sie erklären, warum eine unterschiedliche Behandlung von Männern und Frauen nur selten wahrgenommen wird, und wenn sie doch beobachtet, nicht als Ungleichbehandlung oder Diskriminierung aufgefasst wird. In beiden Argumentationslinien ist Geschlecht keine Komponente sozialer Ungleichheit, sondern entweder irrelevant oder notwendige Differenz. Solche Widerspruchskonstellationen in Bezug auf Geschlecht lassen sich mit Angelika Wetterers These der "rhetorischen Modernisierung" interpretieren. Darin hält sie fest, dass heutzutage zwar einerseits die Geschlechterasymmetrie begründungsbedürftig geworden sei, sich aber gleichzeitig bislang noch kein tiefgreifender sozialer Wandel abzeichne:

„Unsichtbar und aus dem Repertoire dessen, worüber sich sprechen lässt, ausgeschlossen, wird [...] vor allem die hierarchische Struktur der Geschlechterunterscheidung. Die rhetorische Modernisierung schließt als ihre Kehrseite die De-Thematisierung der Ungleichheit zwischen den Geschlechtern ein. Im Prozess der rhetorischen Modernisierung verändert sich nicht nur das Reden über die Geschlechter, sondern auch das Schweigen; verschiebt sich die Grenze zwischen dem, worüber sich sprechen, und dem, worüber sich nur Stillschweigen bewahren lässt." (Wetterer 2005: 77)

Während die Studierenden also einerseits Beispiele von ungleicher Behandlung von Frauen und Männern vorbringen können, verstehen sie diese nicht als systematische Ungleichheit oder Diskriminierung, sie fallen also unter die „Kehrseite“ der nicht-thematisierbaren Ungleichheitsdimension in dieser Lehrveranstaltung zur Ungleichheit an der Universität. Die These Wetterers, dass „Reproduktionsweisen der Ungleichheit indirekter und subkutaner geworden" (Wetterer 2005: 88) sind, lässt sich also auch im Rahmen der Lehrer_innenbildung bestätigen, etwa dann, wenn man auf das Thema Klasse blickt.

\subsection{Klasse - das Ideal des Bildungsbürgertums}

Wie bereits von anderen Autor_innen festgestellt wurde, lässt sich im Rahmen unserer Lehrveranstaltung eine vergleichsweise höhere soziale Durchlässigkeit im Lehramtsstudium feststellen, als es etwa in Studienrichtungen wie Medizin oder Jus der Fall ist (Rothland 2011). Ein Blick auf die in der Lehrveranstaltung erhobenen Daten zeigt deutlich, dass Lehramtsstudierende zu einem überwiegenden Anteil aus Familien kommen, in welchen Vater und Mutter entweder über einen Lehrabschluss oder über Matura, nicht aber über einen Hochschulabschluss verfügen. Entgegen unserer ersten Eindrücke aus anderen Lehrveranstaltungen kommt dabei nur ein sehr geringer Anteil der Lehramtsstudierenden aus Familien, in denen die Eltern selbst als Lehrer_innen tätig sind oder waren. Dieser Eindruck verfestigt sich in den Erzählungen der interviewten Studierenden über ihre Anfangsschwierigkeiten auf der Hochschule und über ihre Erfahrungen, als erstes Familienmitglied überhaupt mit Universität in Berührung zu kommen. Ein Studierender beschreibt seinen Einstieg an der Uni etwa folgendermaßen: „Nein, für mich war ehrlich gesagt alles komplett neu, weil vor 
mir in meiner Familie war noch keiner auf einer Universität und ich bin der Erste, der sich inskribiert hat." (SU1_05: 11) Und auch ein weiterer Studierender stellt sich im Rahmen einer Reflexion die folgende Frage: „Aus meiner Familie hat sonst niemand die Matura geschafft, wie soll ich das Studium ganz allein stemmen?" (LW)

Wenngleich also das akademische Feld für viele der Lehramtsstudierenden etwas Neues ist, so wird dennoch von den Studierenden selbst das Ideal des Bildungsbürgertums hochgehalten, wie sich vor allem in einer Übung zur Selbstreflexion in Bezug auf Wissenschaft zeigt. Aus dem Material geht hervor, dass der Stellenwert von Wissen (und generell kulturellem Kapital) in den Familien der Lehramtsstudierenden als (sehr) hoch beschrieben wird und das Elternhaus Bemühungen zeigt, seinen Erwerb auf verschiedene Weisen zu fördern. Dies lässt sich vor allem an Erzählungen über die Fülle an Büchern - einmal sogar den Besitz einer Bibliothek -, den Konsum von Wissenssendungen, die Anschaffung von Musikinstrumenten und die Finanzierung von Musikunterricht oder den Besuch von kulturellen Veranstaltungen festmachen. Bezogen auf das Zugehörigkeitsgefühl an der Universität scheinen Schwierigkeiten - mit Ausnahme von Orientierungsproblemen - im Setting der Lehrveranstaltung nicht äußerbar, vielmehr wissen die Studierenden, „wie der Hase läuft", denn es würden an der Uni vor allem jene Leute aufeinandertreffen, die es geschafft haben: „Wir sind schon gefiltert", so Studierende im Rahmen der Reflexion zur Übung "Ich und die Wissenschaft“."

Es war vielen Studierenden im Rahmen von Diskussionen während der Lehrveranstaltung sichtlich unangenehm, über ihre eigene soziale Herkunft und die damit verbundenen Privilegien oder Mechanismen der Ausgrenzung zu sprechen. Sie stören sich etwa auch an Begrifflichkeiten und vor allem der Zuordnung zur Arbeiter_innenklasse, zur Bildungselite, zu Akademiker_innenhaushalten etc. Dies äußert sich etwa in einer hitzigen Diskussion darüber, warum denn Kinder aus der Arbeiter_innenklasse nicht die gleichen Zugänge zu Bildung hätten, könne man doch auch als Arbeiter_innenkind einer reichen Familie entstammen.

Die Wichtigkeit des Nachdenkens über den Zusammenhang von sozialer Herkunft und Bildung verdeutlicht sich augenfällig in einem Interviewausschnitt, in welchem ein Lehramtsstudierender aus einer

11 Angelehnt an den "Leitfaden zur Selbstreflexion: Ich und die Wissenschaft" in Costa/Mendel 2018:11. sogenannten Arbeiter_innenfamilie von seinem durch ökonomisches Kapital beeinflussten Bildungsweg folgendermaßen erzählt:

„Und bei meinem ersten Jahr Sekundarstufe 1 haben meine Eltern sich das nicht mehr leisten können [bezieht sich auf eine private Schule] und ich bin dann auf eine Sport-Hauptschule gekommen. [...] da war ich irgendwie der Outsider, weil ich der Einzige in der Klasse war, der gern lernt und der sich fasziniert für neues Wissen." (SU1_o8: 154-156)

Obwohl sich dieser Zusammenhang zwischen Kapitalien und Bildungserfolg in den Erfahrungen Einzelner widerspiegelt, werden diese Erfahrungen auch hier nicht als Ausdruck struktureller Ungleichheit gewertet, sondern häufig als individuelles Problem verhandelt.

\subsection{Kollektive Erfahrungen und kritische Refle- xion des Habitus}

Diesen Fokus auf individuelle Problematiken sozialer Ungleichheit zu überwinden, bedeutet für uns als Lehrveranstaltungsleiter_innen stets aufs Neue darauf zu verweisen, über den eigenen Tellerrand zu blicken. Dies gelingt in einigen Übungen etwas besser, insbesondere in der Bearbeitung der Themen rund um die Erinnerungsarbeit, welche nicht nur gemeinsame Erfahrungen von Studierenden sichtbar und damit diskutierbar macht, sondern ebenso den Anspruch erhebt, das Alltagsdenken zu dekonstruieren und gewohnte Normalitätsvorstellungen zu hinterfragen. Dies passiert vor allem durch die Identifikation von Klischees und Selbstverständlichkeiten, auf die wir beim Erzählen über uns selbst zurückgreifen. In der Analyse und Interpretation von Erinnerungsgeschichten zum Thema „Als ich mich dazu entschieden habe, Lehrer/in zu werden“ einer studentischen Kleingruppe zeigte sich, dass in allen Texten auf dasselbe „Klischee“ (oder dieselbe Selbstverständlichkeit) zurückgegriffen wird, nämlich dass sich die Eignung dafür „immer schon" gezeigt hat. Es wird in allen Texten als eine Art Hineinwachsen in eine Rolle beschrieben, mit der man schon immer geliebäugelt hat: „Bereits als Kind spielte sie gerne die strenge Lehrerin“ (Akinom). Es wird von Vorbildern, Interessen und Hobbys berichtet („Es bereitete ihm eine große Freude zu sehen, wie interessiert und glücklich die Kinder darüber waren, eine weitere Person vor sich stehen zu haben, die sich um sie kümmert." (Seki)), aber in allen Texten besteht die Lücke darin, dass überhaupt nicht klar und nach- 


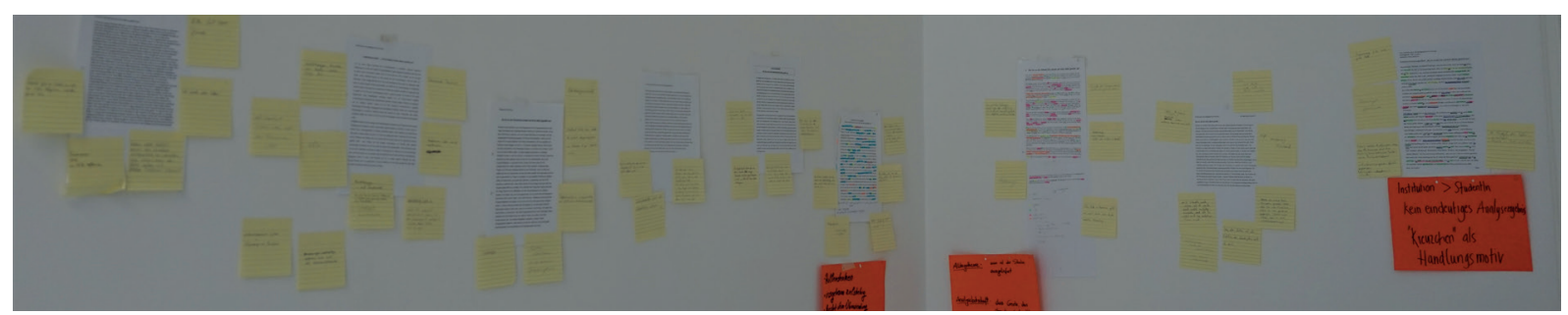

Quelle: Eigene Fotodokumentation der Lehrveranstaltung im WiSe 2018/19

vollziehbar wird, warum es zu dieser Studienentscheidung kam. Themen wie Autorität und Führung oder die Liebe zum Erklären tauchen auf und scheinen gesellschaftliche Diskurse über den_die „ideale_n Lehrer_in“ zu repräsentieren: „Ihm war klar, dass er für andere Menschen immer sehr offen war und auch schon im Fußball, wo er bereits Jugendmannschaften mittrainierte, auch einen guten Umgang mit Kindern zeigte und da auch sofort einen gewissen Respekt von ihnen erlangte." (Eichi).

Gerade weil das eigene Denken in Klischees durch die Erinnerungsarbeit so schonungslos offengelegt wird, hat die Methode das Potenzial, eigene eingefahrene Denkweisen ein Stück weit zu irritieren. Neben der Irritation ermöglicht es die Erinnerungsarbeit aber auch, strukturelle Mechanismen von Ungleichheit stärker in den Fokus zu rücken und emanzipatorische Perspektiven zu entwerfen. Dies zeigte sich beispielsweise in der Auseinandersetzung der Lehrveranstaltungsgruppe mit dem Thema „Als ich an der Uni einmal auf mich allein gestellt war“. Die Analyse der Geschichten brachte hervor, dass Studierende besonders oft vor dem Problem stehen, dass die Regeln an der Universität andere sind, als sie glauben. Es scheint ungeschriebene Gesetze zu geben, die Stress erzeugen, wenn man sie nicht kennt: „Warum wusste er nichts davon, warum hatten ihm seine Studienkolleginnen und -kollegen nichts davon gesagt, dass in dieser Prüfung das Abschreiben offensichtlich üblich war?" (Totoro), „Aber warum hatte er eine Fünf.“ (Elias), „Nicht wissend, dass dies ohnehin keine Anwesenheitspflicht mit sich brachte, bekam er langsam Stress, das richtige Gebäude zu finden, da er nicht am ersten Tag zu spät kommen wollte." (Gernie). Die Studierenden fühlen sich auf sich allein gestellt, gleichzeitig aber für die eigene Leistung verantwortlich. Der Anspruch, es alleine schaffen zu müssen, bringt die Angst vor dem Scheitern mit sich. Die gemeinsame Verständigung über diese scheinbar individuellen Unzulänglichkeiten und Ängste kann das Bewusstsein dafür stärken, mit den eigenen Erfahrungen nicht alleine dazustehen, und den Blick weg von individuellen hin zu kollektiven Erfahrungen lenken. Ganz konkret werden im letzten Arbeitsschritt der Erinnerungsarbeit gemeinsame Erfahrungen benannt und den bearbeiteten Geschichten hinzugefügt (z. B. per Haftzettel dazugeklebt).

Die Methode visualisiert und macht daher tatsächlich greifbar, dass die Einzelnen mit ihren Erfahrungen nicht alleine sind. Damit stärkt sie deren Möglichkeiten, sich auch gemeinsam über Erlebtes zu verständigen, sich über Veränderungsmöglichkeiten auszutauschen und gestärkt aus diesem Reflexions- und Austauschprozess hervorzugehen.

\section{Fazit}

Mit unserem Forschungsprojekt haben wir uns zum Ziel gesetzt, Lehramtsstudierenden Einsichten über soziale Ungleichheit und die eigene Verortung in der Gesellschaft zu ermöglichen und diese mithilfe vielfältiger Übungen und Methoden kritisch zu reflektieren. Dies soll Studierende dazu anregen, Habitusreflexivität zu entwickeln, um die durch das Schulsystem nahegelegten Reproduktionsmechanismen sozialer Ungleichheit nicht ganz unwidersprochen zu unterstützen. Wir zielen also darauf ab, das eigene Handeln und die eigenen Wertevorstellungen vor dem Hintergrund gesellschaftlicher Machtverhältnisse $\mathrm{zu}$ begreifen. Damit wird auch eine kritische Sicht auf das System (Hoch-)Schule erzeugt, die die Lehramtsstudierenden jedoch nicht demotivieren, sondern Transformationspotenziale aufzeigen soll.

Als besonders produktiv haben wir die Auseinandersetzung mit spezifisch studentischen Erfahrungen erlebt, die sich aus der fehlenden Passung zwischen individuellem Habitus und den Erwartungen der Universität ergeben. Gerade hier besteht für alle Studierenden die Herausforderung darin, sich neu zu orientieren und zurechtzufinden. Auch wenn das einigen Studierenden weitaus besser gelingt - sie also den pas- 
senden Habitus bereits mit Studienbeginn mitbringen -, so teilen doch alle ähnliche Erfahrungen, die sich vermutlich auch durch die stärker verschulte Studienarchitektur, den Prüfungsdruck und die große Distanz zwischen Wissenschaft und Alltagswissen ergeben.

Potenziale des Projektes liegen in der kollektiven Erforschung studentischer Erfahrungen mit Privilegierung und Ausgrenzung und der Auseinandersetzung mit Widerstandsmöglichkeiten. Darüber hinaus braucht es auch im Rahmen des Lehramtsstudiums einen Ort, an dem soziale Herkunft thematisiert und sowohl auf der wissenschaftlichen wie auf der selbstreflexiven Ebene bearbeitet werden kann. Losgelöst vom Lehrveranstaltungskontext ist es daher wichtig, Materialien zu sammeln, (weiter) zu entwickeln und diese für Lehrende und Studierende zugänglich zu machen. Sowohl Lehrer_innen an der Schule als auch Lehrende an der Universität profitieren davon, Habitusreflexivität zu entwickeln, um so sensibel für soziale Ungleichheit zu werden.

\section{Literatur:}

Alkemeyer, Th./Rieger-Ladich, M. (2008): Symbolische Gewalt im pädagogischen Feld: Überlegungen zu einer Forschungsheuristik. In: Schmidt, R./Woltersdorff, V. (Hg.): Symbolische Gewalt. Herrschaftsanalyse nach Pierre Bourdieu. Konstanz: UVK, 103-124.

Aulenbacher B./Meuser, M./Riegraf, B. (2010): Soziologische Geschlechterforschung. Eine Einführung. Wiesbaden: VS.

Bacher, J. (2006): Soziale Ungleichheit, Schullaufbahn und Testleistungen. In: Suchan, B./Wallner-Paschon, Ch./ Schreiner, C. (Hrsg.): PIRLS 2006: Die Lesekompetenz am Ende der Volksschule - Österreichischer Expertenbericht. Graz: Leykam. S.79-101. Online: https://www.bifie. at/wp-content/uploads/2017/o9/PIRLS2006_NEB_Web. pdf [27.8.2019].

Bitschnau, K./Hinteregger, M. (2001): Kollektive Erinnerungsarbeit. In: Hug, T. (Hg.): Wie kommt Wissenschaft $z u$ Wissen? Band 2. Einführung in die Forschungsmethodik und Forschungspraxis. Baltmannsweiler: Schneider Verlag Hohengehren, 342-355.

Bock, M. (1992): Das halbstrukturierte-leitfadenorientierte Tiefeninterview. In: Hoffmeyer-Zlotnik, J. H.P. (Hg.): Analyse verbaler Daten. Über den Umgang mit qualitativen Daten. Opladen: Westdeutscher Verlag, 90-109.

Bohnsack, R./Przyborski, A./Schäffer, B. (2006) (Hg.): Das Gruppendiskussionsverfahren in der Forschungspraxis. Opladen: Barbara Budrich.

Bourdieu, P. (1976): Entwurf einer Theorie der Praxis. Auf den ethnologischen Grundlagen der kabylischen Gesellschaft. Frankfurt am Main: Suhrkamp.
Bourdieu, P. (1997): Die feinen Unterschiede. In: Steinrücke, M. (Hg.): Die verborgenen Mechanismen der Macht. Hamburg: VSA Verlag, 31-47.

Bourdieu, P. (2018): Schriften: Bd. 10: Bildung. Schriften zur Kultursoziologie 2. Berlin: Suhrkamp.

Bourdieu, P./Passeron, J.-C. (1971): Die Illusion der Chancengleichheit. Untersuchungen zur Soziologie des Bildungswesens am Beispiel Frankreichs. Stuttgart: Klett.

Brandes, H. (2002): Der männliche Habitus. Band 2. Männerforschung und Männerpolitik. Opladen: Leske/Budrich.

Brandmayr, M. (2017): Lernen und die gesellschaftliche Reproduktion: Vier Thesen über die Wirksamkeit von Ideologie in der Schule. Momentum Quarterly, 6 (3), 187-202.

Breit, S./Schreiner, C. (2017): Ausgangslage in Österreich Fakten zur Bildungsgerechtigkeit. Schulheft, 42 (4), 7-16.

Castro Varela, M. (2014): Bildungsprivilegien für alle! Interview von migrazine.at. Online: http://www.migrazine.at/ artikel/bildungsprivilegien-f-r-alle [24.08.2018].

Crenshaw, K. (1989): Demarginalizing the Intersection of Race and Sex: A Black Feminist Critique of Antidiscrimination Doctrine, Feminist Theory and Antiracist Politics. University of Chicago Legal Forum, 1 (8), 139-167. Online: http://chicagounbound.uchicago.edu/uclf/ vol1989/iss1/8 [11.8.2018].

Costa, Rosa/Mendel, Iris (2018): Tatsächlich. Feministische Zugänge zu Wissenschaft vermitteln. Lehrbuch. 2, leicht überarbeitete Auflage. Wien: Universität Wien.

El-Mafalaani, A. (2017): Transformationen des Habitus. Praxeologische Zugänge zu sozialer Ungleichheit und Mobilität. In: Rieger-Ladich, M./Grabau, Ch. (Hg.): Pierre Bourdieu. Pädagogische Lektüren. Wiesbaden: Springer, 103-127.

Eribon, D. (2016): Rückkehr nach Reims. Berlin: Suhrkamp.

Eribon, D. (2017): Gesellschaft als Urteil. Klassen, Identitäten, Wege. Berlin: Suhrkamp.

Erler, I. (2011): Bildung - Ungleichheit - symbolische Herrschaft. In: Erler, I./Laimbauer, V./Sertl, M. (Hrsg.): Wie Bourdieu in die Schule kommt. Analysen zu Ungleichheit und Herrschaft im Bildungswesen. Innsbruck: Studienverlag, 22-36.

Friebertshäuser, B./Rieger-Ladich, M./Wigger, L. (2009) (Hg.): Reflexive Erziehungswissenschaft. Forschungsperspektiven im Anschluss an Pierre Bourdieu. Wiesbaden: Springer VS.

Haug, F. (1999): Vorlesungen zur Einführung in die Erinnerungsarbeit. Hamburg. Argument.

Haug, F. (2003): Lernverhältnisse. Selbstbewegungen und Selbstblockierungen. Hamburg: Argument.

Heim, C./Lenger, A./Schumacher, F. (2009): Bildungssoziologie. In: Fröhlich, G./Rehbein, B. (Hg.): Bourdieu-Handbuch. Leben - Werk - Wirkung. Stuttgart/Weimar: J.B. Metzler, 254-263.

Helsper, W. (2018): Lehrerhabitus. In: Paseka, A./KellerSchneider, M./Combe, A. (Hg.): Ungewissheit als Her- 
ausforderung für pädagogisches Handeln. Wiesbaden: VS Springer, 105-140.

Herrmann, K. (2016): Zur Komplizenschaft der Kritik. Eine bildungstheoretische Analyse des widersprüchlichen Involviertseins von Kritik. Momentum Quarterly, 5 (2), $112-120$.

Hipfl, B. (1997): Vom Text zu seinen Lesarten. Analyse von Medienerfahrungen mit Erinnerungsarbeit. Medienimpulse. Beiträge zur Medienpädagogik, 5 (19), 47-58.

Hofbauer, J. (2004): Distinktion. Bewegung an betrieblichen Geschlechtergrenzen. In: Pasero, U./Priddat, U. (Hg.): Organisationen und Netzwerke. Der Fall Gender. Wiesbaden: VS Verlag für Sozialwissenschaften, 45-64.

Hofbauer, J./Krell, G. (2011): Intersektionalität und Diversity mit Bourdieu betrachtet. In: Smykalla, S./Vinz, D. (Hg.): Intersektionalität zwischen Gender und Diversity. Münster: Westfälisches Dampfboot, 96-92.

Klinger, C. (2003): Ungleichheit in den Verhältnissen von Klasse, Rasse und Geschlecht. In: Knapp, G.-A./Wetterer, A. (Hg.): Achsen der Differenz. Gesellschaftstheorie und feministische Kritik II. Münster: Westfälisches Dampfboot, 14-48.

Knoblauch, H. (2003): Habitus und Habitualisierung. Zur Komplementarität von Bourdieu mit dem Sozialkonstruktivismus. In: Rehbein, B./Saalmann, G./Schwengel, H. (Hg.): Pierre Bourdieus Theorie des Sozialen. Probleme und Perspektiven. Konstanz: UVK Verlag, 187-201.

Koller, H.-Ch. (2009): Grundbegriffe, Theorien und Methoden der Erziehungswissenschaft. Eine Einführung. Stuttgart: Kohlhammer.

Kramer, R. (2013): Kulturelle Reproduktion und symbolische Gewalt. Pierre Bourdieus Beitrag zur Bildungssoziologie. Enzyklopädie Erziehungswissenschaft Online. Weinheim: Juventa Verlag.

Lamnek, S. (2005): Gruppendiskussion. Theorie und Praxis. Weinheim: Beltz Verlag.

Liebau, E. (2011): Was Pädagogen an Bourdieu stört. In: Erler, I./Laimbauer, V./Sertl, M. (Hg.): Wie Bourdieu in die Schule kommt. Analysen zu Ungleichheit und Herrschaft im Bildungswesen. Innsbruck: Studienverlag, 10-21.

Liebau, E. (2014): Habitus. In: Wulf, Ch./Zirfas, J. (Hg.): Handbuch Pädagogische Anthropologie. Wiesbaden: Springer, 155-164.

Mayrhofer, L./Oberwimmer, K./Toferer, B./Neubacher, M./ Freunberger, R./Vogtenhuber, S./Baumegger, D. (2019): Indikatoren C: Prozesse des Schulsystems. In: Oberwimmer, K./Vogtenhuber, S./Lassnigg, L./Schreiner, C., (Hg.): Nationaler Bildungsbericht Österreich 2018, Band 1. Das Schulsystem im Spiegel von Daten und Indikatoren. Graz: Leykam: 123-196.

Moebius, S./Wetterer, A. (2011): Symbolische Gewalt. Österreichische Zeitschrift für Soziologie, 36, 1-10.

Müller, U. (1984): Gibt es eine „spezielle“ Methode in der Frauenforschung? In: Zentraleinrichtung zur Förderung von Frauenstudien und Frauenforschung an der Freien
Universität Berlin (Hg.): Methoden in der Frauenforschung. Frankfurt am Main: Fischer, 29-50.

OECD (2018): Bildung auf einen Blick 2018, OECD Indikatoren. Online: https://www.oecd-ilibrary.org/ docserver $/ 6001821$ lw.pdf? expires $=1561105021 \& \mathrm{id}=\mathrm{id} \& \mathrm{ac}$ cname $=$ oidoo6727\&checksum $=$ F52114008D880DE5096 $\mathrm{E}_{3} \mathrm{DE}_{1} \mathrm{BC} 5 \mathrm{OE} 28 \mathrm{~A}$ [21.6.2019].

Ortner, R./Thuswald, M. (2012): In Differenzen schreiben. Kollektive Erinnerungsarbeit zu pädagogischen Situationen. In: Ortner, R. (Hg.): exploring differences. Zur Vermittlung von Forschung und Bildung in pädagogischer Praxis. Wien: Löcker, 65-81.

Rieger-Ladich, M. (2017): Emanzipation als soziale Praxis. Pierre Bourdieu in der Kritik - und ein Versuch, ihn weiterzudenken. In: Rieger-Ladich, M./Grabau, Ch. (Hg.): Pierre Bourdieu. Pädagogische Lektüren. Wiesbaden: Springer VS, 335-362.

Rosenthal, G./Loch, U. (2002): Das Narrative Interview. In: Schaeffer, D./Müller-Mundt, G. (Hg.): Qualitative Gesundheits- und Pflegeforschung. Bern u.a.: Huber Verlag, 221-232.

Rothland, M. (2011): Wer entscheidet sich für den Lehrberuf? Forschung zum soziodemographischen Profil sowie zu Persönlichkeits- und Leistungsmerkmalen angehender Lehrkräfte. In: Terhart, E./Bennewitz, H./Rothland, M. (Hg.): Handbuch der Forschung zum Lehrerberuf. Münster: Waxmann, 243-267.

Schneickert, Ch. (2013): Illusion der Chancengleichheit. Online: https://www.researchgate.net/profile/Christian Schneickert/publication/290433543_Illusion_der_Chancengleichheit/links/5697b3f308ae1c4279051253/Illusionder-Chancengleichheit.pdf [28.8.2019].

Solga, H./Berger, P./Powell, J. (2009): Soziale Ungleichheit. Kein Schnee von gestern! In: Dies. (Hg.): Soziale Ungleichheit. Klassische Texte zur Sozialstrukturanalyse. Frankfurt am Main: Campus Verlag, 11-45.

Statistik Austria (2018): Erwachsenenbildung 2016-2017, Ergebnisse der Adult Education Survey (AES). Online: http:// www.statistik.at/web_de/services/publikationen/5/index.html?includePage $=$ detailedView\&sectionName $=$ Bil dung $\% 2 \mathrm{C}+$ Kultur\&pubId $=768$ [12.4.2019].

Statistik Austria (2015): Registerbasierte Statistiken. Bildung. Schnellbericht 10.21: Der Einfluss des Elternhauses auf die Schulwahl der Kinder in Österreich - Ein Vergleich der Situation 1981 und 2011. Wien: Statistik Austria. Online: https://www.statistik.at/web_de/services/publikationen/5/ index.html?includePage $=$ detailedView\&sectionName $=$ Bildung\%2C+Kultur\&pubId=705 [10.10.2016].

Vogel, D. (2019): Habitusreflexive Beratung im Kontext von Schule als Beitrag zu einer rationalen-demokratischen Pädagogik. In: Dies. (Hg.): Habitusreflexive Beratung im Kontext von Schule. Ein Weg zu mehr Bildungsgerechtigkeit. Wiesbaden: VS Springer, 233-327.

von Unger, H. (2014): Beispiele der methodischen Umsetzung. In: Dies. (Hg.): Partizipative Forschung. Einführung in die Forschungspraxis. Springer VS: Wiesbaden, $69-83$. 
Wetterer, A. (2005): Rhetorische Modernisierung und institutionelle Reflexivität. Die Diskrepanz zwischen Alltagswissen und Alltagspraxis in arbeitsteiligen Geschlechterarrangements. Freiburger FrauenStudien, 11 (16), 75-96.

Winker, G./Degele, N. (2009): Intersektionalität. Zur Analyse sozialer Ungleichheiten. Bielefeld: transcript Verlag. 